\title{
La cultura física: posibilidades de afectación del cuerpo vulnerable y/o susceptible*
}

\author{
Jesús Ortega Bolaños* \\ Recibido: marzo 6 de 2013 • Evaluado: abril 11 de 2013
}

Aceptado: mayo 20 de 2013

\section{Resumen}

La presente reflexión teórica parte de reconocer la construcción historiográfica del cuerpo desde diversos saberes, disciplinas y experiencias; de las ciencias de la vida con enfoques positivos o neopositivos y de las ciencias sociales con enfoques de corte más interpretativo o comprensivo, que muestran escenarios donde el cuerpo es vulnerable y susceptible (lesionar el organismo o sus circunstancias) y la forma en que podrán ser prevenidos los riesgos, y el concepto se construirá a través de la cultura física y sus distintos componentes y escenarios culturales para proponer abordajes intersectoriales o transectoriales en pro de su comprensión. El escenario de la cultura física estará en construcción a través de un diálogo de saberes y relaciones entre lenguajes técnicos y saberes populares que por medio de un intercambio social de conocimientos y prácticas sociales permita disminuir lo vulnerable o lo susceptible de los cuerpos aportando a proyectos de vida y bienestar.

Palabras clave: cultura física, cuerpo vulnerable, susceptibilidad.

" Investigación realizada para proyecto de investigación doctoral Concepciones de justicia del sistema de salud colombiano.

*" Médico Cirujano, Especialista en Epidemiologia, Magister en Salud Pública. Aspirante Doctorado Ciencias Sociales. Docente Investigador, División de Salud, USTA, (Colombia). Correo electrónico: jesusortega@usantotomas.edu.co 


\section{The Physical Culture: affectation possibilities of vulnerable and/or susceptible body}

\section{Abstract}

The present theoretical reflection part of recognize the historiographical construction of the body from several knowledges, disciplines and experiences from the life sciences, social sciences or from cutting interpretive show scenarios where the body is vulnerable and susceptible (damage the body or its circumstances) being this category the way that it could be prevented, constructed or recovered from the physical culture and its different components and cultural scenarios in order to propose intersectorial or cross-sectoral tackles on behalf of its understanding. The Physical Culture scenario would be under construction by a knowledge dialogue and relationships between technical language and popular knowledge and through a social exchange would address the body since elements of physical culture and with human right to construct their life project socially.

Keywords: Physical Culture, vulnerable body, susceptibility. 


\title{
Introducción
}

\author{
"Porque así como el cuerpo es uno, \\ $\mathrm{y}$ tiene muchos miembros, \\ pero todos los miembros del cuerpo, \\ siendo muchos, son un solo cuerpo..." \\ $1^{\circ}$ Carta de San Pablo a los Corintios 12,12-26.
}

El cuerpo, con diversidad de necesidades de cuidado y de salud, así como de construcciones sociales, antropológicas, sociológicas y culturales, ha mostrado desde la colonia que al estar constituido por una diversidad de elementos en interacción con los contextos, explicita ciertas vulnerabilidades y susceptibilidades que deben ser abordadas. Por lo tanto, la cultura física esa una opción de análisis teórico-conceptual, que en su alcance podría brindar una serie de pistas para disminuir la dinámica vulnerable del cuerpo para optimizar las capacidades y oportunidades de construir un proyecto de vida.

\section{Transformaciones históricas del cuerpo}

Nuestro cuerpo es el ejemplo más destacado de lo ambiguo William James

Los acercamientos históricos al cuerpo han mostrado una serie de abordajes desde la higiene privada y la salubridad iniciada en la antigüedad, la mitología, los cuerpos en movimiento, la acción física en las olimpiadas del mundo grecorromano, la higiene pública, hasta llegar al siglo XV, la modernidad muestra otras formas de ver el cuerpo con el adoctrinamiento y la educación, la mirada foucaultiana sobre la vigilancia y el castigo, y las normas de urbanidad. Y luego, en su configuración de mediados de los años ochenta con el viraje lingüístico que dio paso a la construcción del análisis crítico del discurso en los noventa, se generó el escenario de las voces dominadas 
-feminismo, población vulnerable, entre otras- por discursos hegemónicos $\mathrm{y}$ de mantenimiento de poder.

Dicha construcción histórica del cuerpo y sus acepciones han tenido la intención de responder a las necesidades de supervivencia, adaptación, higiene, sanidad, poder, y de una mirada estética para construir un "modelo de cuerpo", el fenómeno o esencia que desde el punto de vista husseriano, privilegia la conciencia.

Husserl, desde la propuesta fenomenológica considerada como una corriente idealista subjetiva aplicada al cuerpo, describiría la comprensión a partir de la experiencia más originaria posible del mismo, donde este, como fenómeno, sería observado en los sujetos de estudio en busca de su esencia, y durante un fenómeno de reducción de la realidad "epojé". ${ }^{1}$ En este sentido, el cuerpo en el mundo tendría una esfera privada donde la subjetividad y la conciencia es un camino a la acción, siendo una esfera pública a través de la intersubjetividad y el lenguaje, el otro camino para llegar a la misma dirección.

El cuerpo, desde dichos elementos fenomenológicos, podría generar hábitos, acciones promocionales y prácticas sociales, que configurarían una praxis para construir y transformar las acciones planteadas.

El escenario cultural posmoderno muestra dos miradas al cuerpo relacionadas con la construcción social e identitaria, donde el discurso de los cuerpos genera una serie de tensiones e imágenes de poder de unos sobre otros dominados y vulnerados; por otro, lado está la mirada desde la imagen, las identidades no políticas y más cercanas al fitness, el bodybuilding, la anorexia, el ayuno y el manejo estético del cuerpo en un esfuerzo por recuperar dimensiones y vivencias anteriores del cuerpo (Portela, 2001).

Según Barbero (1996: 44), la educación física se ha construido socialmente desde el modelo del hombre-máquina, con el ideal del deporte educativo, mirada que requiere incorporar el contexto para comprender categorías como el cuerpo, razón por la cual se pregunta “ ¿Dónde está la reflexión que nos permita detectar las conexiones entre el arte del cuerpo que enseñamos como legítimo en las clases de educación física, y el contexto social más amplio, con sus relaciones de poder y dominación?”.

1 Es el acto por medio del cual el mundo es colocado en suspenso. Para no generar afectaciones en la descripción del fenómeno a estudiar, esto es" el cuerpo". 
Según Kottow (2009: 52), "lo racional no permea todo el espectro de la experiencia humana, ni da razón del mundo afectivo, o de la transcendencia artística" concentrándose en la búsqueda de lo que es propio de lo humano más allá de lo material, lo cual permite a la mente ejercer una acción hegemónica y un diálogo con el cuerpo más allá de lo cognitivo, permitiendo que las percepciones y los sentimientos se configuren en posibilidades de resignificación de lo humano.

Es claro que el animal sin "atributos racionales" responde desde sus instintos, de la misma manera que el ser humano, desde sus funciones intelectuales, es capaz de elaborar una respuesta más allá del acto reflejo de supervivencia, llevándolo a construir y enaltecer una idea de cuerpo a través de prácticas físicas, y en los últimos tiempos, a la formación de una cultura física. Este escenario de racionalidad, libertad y eticidad del ser humano muestra un cuerpo afectado desde lo externo, lleva a distinciones planteadas por Merleau-Ponty, del cuerpo vivo material y el cuerpo vivido existencial, reflexión que hace en el escenario médico Plugge (1970) citado en Kottow y Bustos (2005).

El escenario dinámico del ser humano muestra la necesidad de un cuerpo sin el cual no se podría existir, objetivamente captado y explorado desde las ciencias de la vida constituyéndose en el "cuerpo vivo". De esta manera, el discurso y la subjetividad del ser humano revela el avance del concepto de cuerpo, relacionado desde las experiencias, es decir "el cuerpo vivido".

Estas observaciones sobre el cuerpo llevaron a Ortega y Gasset "a reflexiones sobre el extracuerpo visible y observable en contraste con el intracuerpo que se vivencia subjetivamente; también Sartre hablo de un cuerpo para todos, cuerpo público y un cuerpo para sí o cuerpo íntimo" (Kottow, 2004: 60).

Múltiples son las interpretaciones y polisemias construidas históricamente desde distintos saberes sobre el cuerpo, donde su connotación de privado y de relación con el otro y otros cuerpos, nos dejará explorar escenarios y categorías del mundo y del sistema social, como la pobreza, el bienestar, el disfrute, lo culturalmente elaborado; de aquí se deprende que la relación entre cultura física y la idea de cuerpo vulnerable resulta de una construcción socio-histórica, política y económica entre seres humanos.

Por lo anterior, se podría plantear que el concepto de cuerpo ha sido reemplazado por los múltiples discursos, lo que ha propiciado diversas 
maneras de entenderlo, teniendo como medio de interpretación el lenguaje, siendo transmisión de conocimiento y experiencia a través de los actos de habla que se realizan de generación en generación y en escenarios sociales particulares, considerando el enfoque de tradición y cultura como contextos que permitan relacionar el cuerpo con la cultura física.

\section{Vulnerabilidad y/o susceptibilidad del cuerpo}

Las relaciones históricas del cuerpo y la cultura física buscan reconocer elementos del entorno social, tales como los contextos políticos, sociales, y económicos que se desarrollan en su devenir, y en relación con el cuerpo per se, el contexto de la vulnerabilidad. Este concepto planteado desde la antigüedad, en el escenario filosófico griego, muestra una atmósfera de comprensión y culto al cuerpo que permite evidenciar relaciones o fracturas del alma con el carácter físico del individuo, en el caso de otros mundos, otras vidas, y la figura de la reencarnación como premio a una evolución o a la bondad/maldad expresada en anteriores cuerpos. Aquí, el cuerpo y el alma se muestran separados: lo visible y lo invisible, lo que está destinado a perder su identidad y lo que siempre la conserva, lo mortal y lo divino, el hombre superior (filósofo) - hombre inferior (el esclavo) quien solo posee un cuerpo, un cuerpo colectivo y del que no interesa saber por su suerte.

Desde la edad media, las ideas platónicas son aceptadas en la educación física y la pedagogía y avanzan en la modernidad, recogiendo hábitos y formas de ver la cultura física del cuerpo. En el caso del pensamiento de Santo Tomás, se muestra la importancia que da a la materia en la constitución del hombre y en el conocimiento, siguiendo a Aristóteles en sus investigaciones biológicas, éticas y morales relacionados con el bien, la virtud, la salubridad, lo sano y toda la polisemia relacionada con la virtud y la cultura física.

En este sentido, Aristóteles (1945: 141) habla de la "fronesis" o prudencia, mediante la cual el médico es capaz de discernir lo correcto y lo bueno al enfrentar una posición moral determinada; plantea analogías como "asimila la salud del cuerpo (medicina) con la salud del alma (filosofía)", por tanto la virtud es planteada como "la mejor disposición, facultad o poder de las cosas en todas las ocasiones en que hay que hacer un uso o una obra cualquiera de estas mismas cosas". 
Kottow (2005) plantea que el sujeto vulnerable es aquél que vive en peligro de ser dañado. Esta definición es tan genérica que se aplica a todos los seres humanos, ya que la vida no está amparada por suficiente vigor biológico sino que, a la inversa, arraiga en la necesidad insoslayable de realizarse como un proyecto de vida que está en permanente riesgo de fracasar. Así lo vio J. H. Herder (1744-1803) al definir al ser humano como una existencia que debe realizarse; sus ideas fueron predecesoras del existencialismo del siglo XX, especialmente en la fórmula sartreana según la cual, la existencia precede a la esencia, y en el modo peculiar de ser del hombre, que Heidegger denominó Dasein. Esta imagen antropológica ha sido retomada por filósofos como Alasdair MacIntyre y Onoora O’Neill, quienes específicamente utilizan el concepto de vulnerabilidad para caracterizar la condición humana de fragilidad.

Siendo condición humana esencial y universal, la vulnerabilidad no tiene modo de ser eliminada, por lo cual desde el siglo XV en Inglaterra, la expedición de ley de pobres orientaba sobre el manejo de protección que debería dársele a las personas que carecían de medios para construir sus capacidades y aprovechar el sistema de oportunidades que tendrían a través de un oficio o un arte. Desde el siglo XVII se ha entendido que la vida en sociedad genera la necesidad y la posibilidad de dar una protección general contra la vulnerabilidad a través de una función de resguardo ejercida por el Estado y contemporáneamente, mediante la proclamación y el respeto de los Derechos Humanos. Como la vulnerabilidad afecta a todos los individuos por igual, corresponde que se otorgue protección, también igualitaria, en concordancia con el principio de justicia.

Los filósofos MacIntyre y O’Neill han tenido la perspicacia de reconocer que, si bien la humanidad entera es vulnerable, hay cohortes de individuos que además han sido lesionados en su organismo o en sus circunstancias, quedando incapacitados para integrarse en la sociedad, asumir las tareas que enfrentan, o hacer uso de las opciones de construir su vida. Estas personas, dañadas y afectadas negativamente, son vulnerables, como todo ser humano, y además, susceptibles o predispuestas a nuevos daños. Los susceptibles quedan a la intemperie, si son tratados como vulnerables: reciben protección general pero no el tratamiento de sus males. Para lograr la asistencia específica que las susceptibilidades requieren, son precisos programas que desarrollen derechos positivos cuyo correlato sean obligaciones asignadas y especificadas. 
En la medicina es posible ilustrar las distorsiones que resultan de no distinguir vulnerables de susceptibles, tanto en escenarios públicos como privados. Desde el Estado asistencialista de Colombia, la vulnerabilidad suscita políticas protectoras sustentadas por un principio de justicia, válidas para todos por igual. En el ámbito sanitario se traduce este resguardo en un derecho a protección de la salud -como lo formula la Constitución de 1991-. De allí derivan algunas obligaciones comunitarias como medicina preventiva, programas de salud pública, protección del entorno social y natural. Aquí, él derecho a la protección de la salud reduce la vulnerabilidad orgánica de toda la ciudadanía en relación con los factores de riesgo, pero no contiene elementos para asistir a los susceptibles sin capacidad de pago, que para la medicina son los enfermos con posibilidades de recuperación primaria de su salud.

El escenario de lo justo en salud, tiene que ver no solo con un principio de justicia, sino con la inequidad del sistema que lo cobija. Por tal razón, al derecho de protección social en salud es necesario agregarle un derecho fundamental a la atención médica en condiciones de igualdad, para lo cual se deben llegar a acuerdos sociales que permitan generar leyes, reglamentos, crear o fortalecer instituciones y convocar personas que se hagan cargo de brindar el tratamiento que los susceptibles requieren, y ejerzan sus posibilidades de representación y participación política para exigir en nombre de los sujetos vulnerables, sus derechos (Foucault, 2007).

\section{La cultura física y su relación con el cuerpo}

"Discursos que sobre el cuerpo elaboran las culturas" Miguel Kottom

Para la antropología médica, el cuerpo humano no es algo sino alguien que vive ese cuerpo con significaciones, funcionamiento, bienestar, relaciones y también con claudicaciones, insuficiencias y disfunciones (Pedraza, 2009). En este sentido, el cuerpo es considerado una caja de resonancia de condiciones del ambiente y de circunstancias sociales, como un suceso o acontecimiento histórico (Pera, 2006). Desde esta perspectiva, lo estético del adorno cultural se considera un medio especializado en la formación y comunicación de la identidad personal y social. Aquí podemos plantear que tanto un 
piercing como un arete tienen y cumplen la misma función, la de perforar la misma parte del cuerpo; lo que cambia es la significación cultural que representan estos objetos para el cuerpo y ante los demás.

La cultura física muestra distintas dimensiones y dinámicas del cuerpo en su ejercicio de interrelación con sus contextos (Berger y Luckmann 1998). En este sentido, el cuerpo subjetivo, desde la visión fenomenológica, plantea la posibilidad de afirmar que la experiencia del cuerpo propio se identifica con los actos humanos, por lo que a partir de Husserl (18591938), el cuerpo es el órgano del YO considerado una entidad intermedia entre el mundo exterior y un espacio temporal particular, y en este desarrollo fenomenológico sobre la integridad del YO, se llega a Merleau-Ponty (1945) quien plantea el ser y tener cuerpo. De esa forma, la naturaleza del cuerpo desde sus inicios como materia, su configuración física y de transformación como cuerpo vivido, las miradas estéticas desde la cultura física y su conocimiento humano requieren de la historia, la corporeidad y las alternativas de la vida subjetiva.

El cuerpo subjetivo se distingue de las estructuras corporales especiales, donde la esencia y el ser como expresiones de la subjetividad son de naturaleza espiritual, se está de acuerdo con que desde la historiografía, en la subjetividad no hay cuerpo en sí; sino que el cuerpo es una entidad simbólica socialmente instituida como resultado de operaciones productoras de sentido sobre la Carne Sapiens, pero el cuerpo es una institución situacional que se construye en el nosotros y con una intencionalidad de configurar un ejercicio de poder y mantenimiento de ideología entre cuerpos que ejercen poder mental sobre otros cuerpos dominados.

Aquí es importante resaltar que la expresión cultural y estética de las estructuras corporales especiales, está relacionada con el bipedismo, la ubicación de los ojos y la mirada, la ubicación de los órganos sexuales para la relación sexual de frente, la asimetría funcional del cerebro, la especialización de las manos y el aparato vocal (Crossley, 1995); se manifiesta en aspectos como la corporeidad de los objetos en experiencias primitivas, encontrados entre otros cuerpos, la vivencia del dolor físico y las necesidades biológicas (Foucault, 1976).

Ser y tener se identifican en el yo subjetivo (Berthelot, 1995); la existencia subjetiva es el mayor bien del hombre que se podría derivar de procesos o modos de subjetivación produciéndose la corrupción de la materia, visión 
que debe ser superada para la coexistencia de la subjetividad y la objetividad del cuerpo. Por lo anterior, no hay algo psíquico o algo somático en la subjetividad, como pueden ser las lágrimas o el ponerse colorado.

A lo largo del escenario presentado de múltiples transformaciones y construcciones del cuerpo vulnerable o susceptible y sus posibilidades de análisis e intervención desde la cultura, las marcas corporales se vuelven cada vez más profundas y menos epidérmicas, incorporando prácticas sociales de cultura del cuerpo que han sido aceptadas y acogidas socialmente, creando subculturas del cuerpo que podrán ser miradas desde la cultura física para tratar de entender e interpretar los comportamientos y el manejo del cuerpo en la actual sociedad de consumo, dentro de un escenario globalizado y orientado desde discursos hegemónicos de poder que privilegian lo superficial y excluyen cuerpos vividos y susceptibles (Kottow, 2004).

La cultura del tacto y la relación, han sido desplazadas por la cultura visual y tecnológica en escenarios de relación virtual, donde lo externo de la cultura y lo que puede ser vivenciado y emulado a través de redes de sociedad virtual no corresponden a un cuerpo real, sino ideal, que impresiona los sentidos y fundamentalmente satura las sensaciones, convirtiéndose en culturas y subculturas que envician y provocan mayor dependencia (Mauss, 1935). Aquí, la visión es la menos corporal de los sentidos, dado que hay múltiples vías de acceso al cuerpo vivido con deformación superficial que privilegia otras formas no corporales de afectación de los sentidos y sus percepciones.

La cultura del cuerpo se va construyendo a partir de considerar el cuerpo como "sujeto" que piensa, sabe, desea, hace, se evalúa y se reconceptúa de acuerdo a distintos intereses, siendo, según Soriano (2009: 115), "una unidad multideterminada, con aspectos cognitivos, instrumentales, tónico emocionales, con poder y querer hacer, el cual se considera una vía y soporte de nuestro mundo conceptual, afectivo, expresivo y de deseo".

La construcción de un campo de conocimiento sobre el cuerpo, a partir de sus diversas aproximaciones e interpretaciones desde lo estético y desde la cultura física, son fundamentales para avanzar en proponer un análisis más integral, donde los elementos teóricos y conceptuales que tienen su expresión de hacer en el movimiento y en la actividad física, nos llevan a reflexionar sobre la categoría cuerpo vulnerable-susceptible y sus diversas 
acepciones de conocimiento a partir de las cuales ha sido planteado (Turner, 1984).

Por tanto, considerar elementos teóricos y conceptuales desde saberes construidos en escenarios sociales donde interactúan cuerpos vulnerables o cuerpos susceptibles que configuran cuerpos en masa, en procura de acercarse a un ejercicio de reconocimiento de sus derechos para ser satisfechas sus necesidades sociales particulares relacionadas con el movimiento y la actividad física, se constituye en el reto que desde la cultura física se debe potencializar.

Un primer elemento a discutir tiene que ver con las diversas expresiones conceptuales de la cultura física; algunas concepciones son asimiladas con la actividad física, otras con la cultura física, entendiéndola como parte de la cultura, representando el conjunto de experiencias y logros obtenidos de la creación y aplicación de principios, fundamentos, condiciones, metodologías orientadas a la ejercitación del ser humano, manifestaciones y realizaciones humanas en materia de actividad física encaminada al ocio, educación, competición, aptitud física y salud.

Plantear en esta discusión que el abordaje desde la cultura va a permitir desarrollar análisis interpretativos a partir de construir inductivamente conocimiento particular sobre la temática de la cultura física, requiere indagar sobre los diferentes enfoques sociales de la cultura, y reflexionar sobre la construcción social del concepto y sus diferencias con la tradición y comunicación de saberes en el tiempo y entre diferentes culturas (Pedraza, 2004).

El cuerpo y el movimiento en su relación fundamental con la actividad física hacia una cultura física (Prieto, Naranjo y García, 2005), muestran una serie de conceptos e interacciones, así:

- El modelo relacionado con la cultura física, según Schütz (1993), podría ser "un conjunto de subsistemas con enfoque comprensivo y una serie de orientaciones y elementos articulados coherentemente, destinados a generar interpretaciones teóricas de la relación movimiento corporal-actividad física-salud". Para Prieto et al. (2005: 49) es un cuerpo con movimiento corporal entendido desde la adaptación y desde la expresión y condición social.

- El movimiento es una categoría que puede tener una armonía en su praxis o una serie de estereotipos, los cuales podrán ser percibidos como desordenados o con una no clara intencionalidad. En este enfoque se 
puede decir que el movimiento no solo articula el cuerpo sino la cultura física en pro de un movimiento que permita una dinámica agradable y estética que al transmitirse podrá ir configurando una cultura (Giddens, 1997).

- La actividad física es entendida como el estado de permanecer en acción y movimiento, acción que al ordenarse como un conjunto de acciones motoras con un objetivo específico se vuelve "ejercicio físico" (Prieto, et al., 2005: 51) y que por tanto, se puede considerar un dispositivo para mejorar la salud.

De esta manera, la actividad física competitiva, educativa o recreativa (Contreras, 1998), y la educación física relacionada con la salud, muestran avances de conocimiento relacionados con enfoques y modelos médicos, psicoeducativos o sociocríticos (Guerrero, 2010); que han avanzado en el funcionamiento del cuerpo y efectos del ejercicio sobre sí mismo, en estilos de vida personal, bienestar de vida y en la crítica social e ideológica sobre el cuerpo y la cultura física (Uribe, s. f.).

\section{Discusión final}

Se han planteado una serie de elementos en la reflexión teórica que permiten mostrar una mirada crítica a los aspectos relacionados con el cuerpo, con su apariencia, su duración, su curación, su funcionamiento y su representatividad simbólica. Algunos autores atribuyen este fenómeno a los trascendentales cambios en su concepción, fruto de lo que ha sido llamada la cultura de la posmodernidad, entendida como la superación del proyecto moderno basado en la razón como instrumento de comprensión de la realidad.

La mirada colectiva del cuerpo plantea la categoría de población con sus categorías intrínsecas como raza, clase, edad, sexo, y formación social y política del biopoder que se manifiesta en las relaciones de ejercicio de poder sobre la población vulnerable. Aquí el discurso higiénico propone al cuerpo como eje de desarrollo de la subjetividad moderna.

En Colombia, el cuerpo se ha considerado por las sociedades de control un instrumento de poder sobre las poblaciones que experimentan el ejercicio de poder viendo vulnerados sus derechos, es el caso de las distintas poblaciones indígenas, afrocolombianas, desplazadas y vulneradas desde las formas económicas de producción y de distribución de la tierra. 
La comprensión del cuerpo como un elemento individual y colectivo, social, cultural y político tiene una orientación analítica fundamental en los estudios transdisciplinares, culturales, donde se plantea el cuerpo como una condición y objeto de biopoder. Se requiere entonces, en su interpretación, del conocimiento basado en lo racional y lo cognitivo, y de percibir la realidad, la interacción social, la vida cotidiana de los sujetos y los colectivos, que en el proceso de aprendizaje, configuran una tradición y una cultura que en escenarios temporales particulares irán constituyendo una cultura física aplicada al cuerpo vulnerable o susceptible.

Por lo anterior, mostrar aspectos fundamentales sobre la transformación del cuerpo a lo largo de la historia, desde diversas disciplinas, ciencias y saberes compartidos, refleja una dinámica de construcción permanente del concepto, así como de una serie de insuficiencias conceptuales sobre el cuerpo, su contexto y su ejercicio biopolítico de poder que se expresa en relaciones de cuerpos dominantes y cuerpos dominados. En este transcender, el cuerpo, se apropia desde su dimensión de vulnerabilidad, que todos los seres humanos experimentan, y asocian con el riesgo, como la susceptibilidad o fragilidad del cuerpo, que ha experimentado cambios ante las exposiciones y presenta pocas posibilidades de recuperar su sentido inicial.

Se puede decir que el escenario de la cultura física se encuentra en construcción, a través de un diálogo de saberes y de las relaciones entre lenguajes técnicos y saberes populares que por medio de un intercambio social puede ser el medio para abordar el cuerpo vulnerable o susceptible que se ha teorizado y planteado en esta discusión. Se propone que el cuerpo puede ser analizado desde aspectos cognitivos, racionalistas, y también desde los sentimientos y las percepciones, ampliando las posibilidades de incidencia de la cultura física sobre el cuerpo con derecho a construir socialmente su proyecto de vida.

\section{Referencias}

Aristóteles. (1945). Moral. La Gran Moral, Moral a Eudemo. Argentina: Colectivo Austral, Espasa Caipe.

Barbero, M. J. (1996). En: Revista de historia de la educación. 311, 13-49.

Berger, P. y Luckmann, T. (1988). La construcción social de la realidad. Barcelona: Herder. 
Berthelot, J.M. (1995). The Body as a Discursive Operator. Or the Aporias of Sociology of the Body. Body \& Society, 1 (1), 13-23.

Contreras, O. (1998). Didáctica de la Educación Física: Un enfoque constructivista. Barcelona: INDE.

Crossley, N. (1995). Merleau-Ponty, the Elusive Body and Carnal Sociology. Body \& Society, 1, 43-63. Recuperado de http://bod.sagepub.com/reports/most-cited

Foucault, M. (2007). Nacimiento de la biopolítica: curso en el college de France 1978- 1979. Buenos Aires: Fondo de Cultura Económica. Traducción: Horacio Pons.

Foucault, M. (1976). La historia de la sexualidad. Barcelona: Ediciones Península.

Giddens, A. (1997). Modernidad e identidad del yo. El yo y la sociedad en la época. Barcelona: Ediciones Península.

Kottow, M. (2004). Vulnerability: What kind of principle is it? Medicine, Health Care and Philosophy, 7, 281-287.

Kottow, M. y Bustos, R. (2005). Antropología médica. Santiago: Editorial Mediterráneo.

Guerrero, F. J. (2010). El cuerpo incierto: corporeidad, tecnologías médicas y cultura contemporánea. Madrid: Consejo Superior de Investigaciones Científicas.

Mauss, M. (1935). Las técnicas del cuerpo. Madrid: J. Crary, y S. Kwinter.

Merleau-Ponty, M. (1945). Phénoménologie de la perception. París: Gallimard.

Pedraza, Z. (2004). El régimen biopolítico en América latina. Iberoamericana, IV (15), 7-19.

Pedraza, Z. (2009). En clave corporal. Conocimiento, experiencia y condición humana. Revista Colombiana de Antropología, 45 (1), 147-168.

Pera, C. (2006). Pensar desde el cuerpo. Ensayo sobre la corporeidad humana. Editorial Tricastela, Madrid. ISBN 84-95840-24-3

Portela, G. (2001). Paradigmas del cuerpo en la educación física. Buenos Aires: Efdeportes.

Prieto, A. Naranjo S. y García L. (2005). Cuerpo-Movimiento: Perspectivas. Bogotá: Centro Editorial Universidad del Rosario.

Soriano, D. (2009). Los caminos de Paulo Freire en Córdoba. Villamarin: Eduvim.

Schütz, A. (1993). La construcción significativa del mundo social. Barcelona: Paidos.

Turner, B. (1984). The Body and Society: explorations in social theory. Oxford: Basil Blackwell.

Uribe, I. D. (s. f). Teoría y práctica de la educación física. Universidad de Antioquia. 